\title{
ASSIS: Assistente Inteligente como Serviço para Plataformas de Ensino a Distância
}

\author{
Francisco Aislan da S. Freitas ${ }^{1}$, Bruno da S. Queiroz ${ }^{1}$, \\ Cassandra R. Joye ${ }^{2}$, Paulo Henrique M. Maia ${ }^{2}$ \\ ${ }^{1}$ BSQ Inova - Soluções Inovadoras - Fortaleza, Ceará, Brasil \\ ${ }^{2}$ Universidade Estadual do Ceará (UECE) Fortaleza, Ceará, Brasil \\ \{aislan, bruno\}@bsqinova.com, \\ projetos.cassandra@gmail.com, pauloh.maia@uece.br
}

\begin{abstract}
School dropout occurs in several teaching modalities, causing losses to everyone involved in the educational process. Mapping, mining and treating student behavior using indicators is possible with Artificial Intelligence techniques integrated in a solution such as ASSIS, an Intelligent Assistant as a service for distance learning platforms. This paper presents how ASSIS was developed, applied and validated to predict potential situations of school dropout using machine learning algorithms using some students' behavioral characteristics on distance learning platforms. The best AI algorithm was the Support Vector Machine, for which the parameters Accuracy, F Score, Recall and Precision obtained as results $95.84 \%, 95.79 \%, 94.74 \%$ and $96.88 \%$, respectively.
\end{abstract}

Resumo. A evasão escolar ocorre em diversas modalidades de ensino, ocasionando perdas à todos envolvidos no processo educacional. Mapear, minerar e tratar o comportamento dos estudantes a partir de indicadores é possível com técnicas de Inteligência Artificial integradas numa solução como o ASSIS ,um ASSIStente Inteligente como serviço para plataformas de ensino a distância. Este artigo apresenta como foi desenvolvida, aplicada e validada uma estrutura para prever situações de potencial de evasão escolar usando como metodologia técnicas de aprendizagem de máquina com características comportamentais dos estudantes nas plataformas EaD. O melhor modelo de IA foi o Support Vector Machine, para o qual os parâmetros Accuracy, F Score, Recall e Precision obtiveram os resultados 95,84\%, 95,79\%, 94,74\% e 96,88\%, respectivamente.

\section{Introdução}

$\mathrm{Na}$ modalidade de educação a distância $(\mathrm{EaD})$, o ensino vai até onde o aprendiz está. Qualquer que seja a plataforma, ele se inscreve, acessa o curso, interage com o conteúdo e com as atividades. O grande problema é que, dos que se inscrevem/matriculam, uma quantidade expressiva não finaliza, evadindo-se do processo. A evasão escolar é um problema recorrente para as instituições de ensino, que têm que continuamente buscar estratégias para reter o estudante.

É possível mapear e detectar comportamentos de potenciais evasões em qualquer modalidade de ensino. Shiratori (2017) declara que existem diversos motivos que contribuem para a evasão escolar, sendo os mais comuns: financeiro, dimensão da instituição, 
número de inscrições da instituição, avaliações acadêmicas, notas e falta de suporte pedagógico. Muitos e diversos são os indicadores de comportamento de potenciais evasores em cursos de EAD. O contexto social, cultural, pedagógico, tecnológico e comercial é propício e oportuno para soluções inovadoras que aumentem a qualidade dos serviços educacionais ao tempo em que melhoram os índices de terminalidade e conclusão dos cursos, combatendo assim riscos de evasão. Nessa via, as tecnologias de Inteligência Artificial (IA) podem efetivamente contribuir para controle e mitigação do problema. A IA já vem sendo aplicada com sucesso em setores como indústria, saúde, segurança, educação e outras áreas [Dourado Jr et al. 2019].

Nesse sentido, este artigo apresenta o ASSIS, um assistente inteligente, disponibilizado como um serviço plugável em ambientes virtuais de aprendizagem (AVA), para fornecer informações para gestores e tutores sobre o risco de evasão dos seus alunos por meio de um sistema web com infográficos e um chatbot. O desenvolvimento do ASSIS e sua validação foi realizado junto a uma instituição parceira que utiliza como AVA o Moodle e possui uma base de 7.200 alunos. Nesse ambiente, o ASSIS foi capaz de realizar predição sobre a evasão de alunos com um índice de acerto de 96\%. Segundo Martinho et al. (2013), utilizando os algoritmos de aprendizagem de máquina, pode-se identificar padrões e recursos que normalmente não são encontrados para modelar a evasão dos alunos. Assim, espera-se que o ASSIS possa contribuir no âmbito educacional, sendo uma ferramenta de predição da evasão escolar com características comportamentais em plataformas $\mathrm{EaD}$, auxiliando os gestores na tomada de decisão para reverter uma possível evasão.

O artigo está dividido em 6 seções, sendo elas: na Seção 2 são descritos os principais trabalhos relacionados, enquanto na Seção 3 são discutidos os algoritmos de aprendizagem de máquina utilizados. Seção 4 aborda a metodologia para criação da ferramenta. Seção 5 apresenta os resultados e, por fim, a seção 6 traz as considerações finais.

\section{Trabalhos relacionados}

Recentemente muitos pesquisadores têm investido em identificar os motivos que levam os alunos a evadir na modalidade EaD [Gütl et al. 2014]. Apesar de existirem diversos recursos de apoio ao estudante para facilitar o aprendizado, a retenção e a conclusão nos cursos e programas de formação, os índices de evasão são ainda recorrentes e alarmantes, necessitando avançar em tecnologias que prevejam e combatam a evas ão.

Existem trabalhos sobre predição da evasão escolar utilizando algoritmos de aprendizagem de máquina em apenas informações acadêmicas Al-Shabandar et al. 2017]|[Xing and Du 2019], gerando modelos de predição que normalmente não são atribuídos como uma funcionalidade em uma interface web intuitiva. A falta desse recurso pode impossibilitar o uso por pessoas que não são experientes na área [Martinho et al. 2013]. O ASSIS proporciona essa funcionalidade.

Já no trabalho proposto por Beltran et al. (2019), os pesquisadores desenvolveram uma plataforma de predição do risco de evasão utilizando algoritmos de aprendizagem de máquina como Naive Bayes, MultiLayer Perceptron e AdaBoost. No entanto, os atributos selecionados para construir o vetor de características são de cunho social, como sexo, idade, estado civil, escola de origem, dentre outros. A plataforma proposta pelos autores reúne essas informações para apresentar os alunos que estão em grupos de risco de evasão, 
dividindo-os em baixo, médio e alto risco. O ASSIS amplia os indicadores para minerar comportamentos e propor ações preventivas e corretivas.

Já a pesquisa desenvolvida por Ramos et al. (2018) apresenta um estudo comparativo de algoritmos de aprendizagem de máquina na previsão da evasão de alunos em EaD. O trabalho utiliza os algoritmos Árvore de decisão, SVM, k-Nearest Neighbors e Regressão Logística. Em relação aos atributos selecionados para essa predição, foram escolhidas as características que envolvem autonomia, diálogo e estrutura. O ASSIS também analisou e experimentou diversos algoritmos a fim de construir um modelo de predição.

Com base nesses e em outros trabalhos, desenvolveu-se um sistema de predição da evasão escolar, retratando os indicadores e métricas mapeados a partir das principais informações comportamentais dos alunos tanto em plataformas $\mathrm{EaD}$ em geral, quanto na da instituição em estudo, permitindo aos gestores realizar as ações ou investigações necessárias para reter a evasão escolar.

\section{Algoritmos de aprendizagem de máquina}

A classificação do risco de evasão utilizando algoritmos de aprendizagem de máquina é realizada com as características comportamentais dos alunos extraídas do AVA da instituição. Isso é feito por meio de um plugin, que envia essas informações para a plataforma ASSIS. Nesta seção, são apresentados os 3 algoritmos de aprendizado de máquina que compõem as simulações computacionais.

\subsection{Perceptron}

Segundo Haykin (2008), o algoritmo Single Layer Perceptron (conhecido por perceptron simples) é capaz de identificar se o problema estudado é linearmente separável ou não. O processo de aprendizagem dessa rede neural é inspirada em redes neurais biológicas: são fornecidos valores de entrada, a rede processa e retorna uma resposta, sendo o neurônio ativado somente se a o valor for maior que um determinado limiar.

\subsection{Regressão Logística}

A Regressão Logística (RL) é uma técnica estatística que visa produzir, a partir de um conjunto de análises, um modelo que permite a previsão de valores obtidos por uma variável categórica, geralmente binária, a partir de uma série de variáveis contínuas ou binárias. O RL é amplamente utilizado nas ciências médicas e sociais e tem outras denominações, como modelo logístico, modelo logit e classificador de entropia máxima [Menard 2002].

\subsection{Support Vector Machines}

O Support Vector Machine (SVM) geralmente pode ser usado para três questões diferentes, dependendo da saída desejada: classificação binária, classificação multiclasse e regressão [Guenther and Schonlau 2016]. O objetivo desse algoritmo é encontrar um hiperplano capaz de separar as classes presentes no problema. Os SVMs podem ter kernels diferentes, dependendo do tipo de problema, como linear, não linear, polinomial, kernel Gaussiano, função de base radial (RBF), sigmoide, dentre outros. 


\section{Metodologia de Desenvolvimento do ASSIS}

Nesta seção, será apresentada a metodologia utilizada para desenvolver o sistema proposto para prever a evasão dos alunos com base nos dados comportamentais na plataforma EaD Moodle. Foram seguidas as seguintes etapas, conforme ilustrado na Figura 1: Aquisição dos dados, Pré-processamento dos dados, Arquitetura da plataforma, Interface Gráfica e Definição de métricas. Essas etapas são detalhadas a seguir.

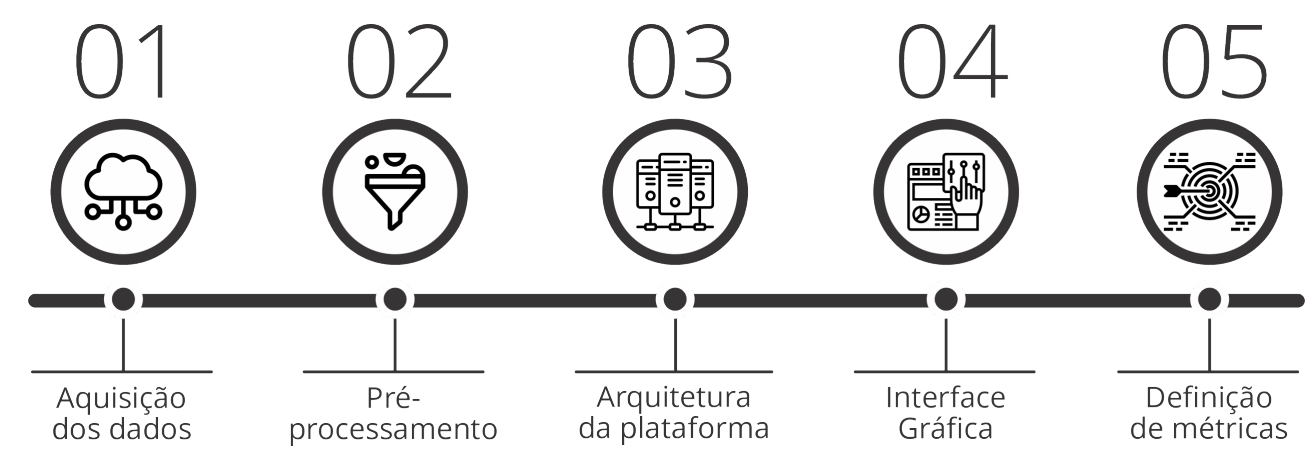

Figura 1. Fluxograma da abordagem proposta para detecção e classificação do perfil do evasor

\subsection{Aquisição dos dados}

Para obter os registros comportamentais dos alunos da instituição alvo, foi criado um plugin que extrai informações do sistema de banco de dados do Moodle. Os dados comportamentais foram armazenados no formato Comma Separated Values (CSV).

A análise foi realizada considerando 58 cursos que envolvem áreas como gestão, finanças e informática, dentre outras. As informações são de uma base histórica das turmas de 2018 a 2020 que já foram encerradas. No total, existem 5360 registros, dos quais 4620 são alunos que concluíram o curso e 740 são evasores.

A seleção dos recursos foi realizada com base nos dados comportamentais dos alunos na plataforma moodle, fornecidos no $\log$ de acesso. Com esses dados, é possível construir um modelo que possa auxiliar a tomada de decisão do gerente na tentativa de reverter a possível situação de evasão do novo aluno nos cursos em andamento. As características foram selecionadas e agrupadas em: (i) Tempo em horas online na plataforma moodle que é o tempo que o aluno passa em cada objeto de aprendizagem (OA); (ii) Persistência que é a quantidade de vezes que o aluno tenta resolver os OAs; (iii) Precedência que é o tempo em horas que o aluno se antecede para resolver as atividades agendadas; e (iv) Desempenho que é mensurado pela nota do aluno nas atividades avaliativas. Ressaltese que a validação da evasão escolar foi obtida por meio da base histórica da instituição.

\subsection{Pré-processamento dos dados}

Os dados selecionados contêm apenas variáveis do tipo contínua, que compõem o vetor de entrada. Já o rótulo, que é utilizado como alvo da classificação é binária, associa on números 0 e 1 aos alunos concludentes e evasores, respectivamente. A Tabela 1 apresenta as variáveis contínuas usadas, bem como os valores máximo e mínimo que eles podem assumir. 
IX Congresso Brasileiro de Informática na Educação (CBIE 2020)

Anais do XXXI Simpósio Brasileiro de Informática na Educação (SBIE 2020)

\begin{tabular}{llcc} 
Tabela 1. Variáveis contínuas com os valores mínimo e máximo cor \\
observados. & \multicolumn{3}{c}{} \\
\cline { 2 - 4 } & Variáveis Contínuas & Mínimo & Máximo \\
& Tempo & 0 horas & 128 horas \\
& Persistência & 0 & 35 \\
Precedência & 0 horas & 200 horas \\
& Desempenho & 0 & 10 \\
\hline
\end{tabular}

Os atributos tempo e precedência são extraídos para a plataforma do ASSIS em horas.Já o atributo persistência é uma variável do tipo inteira, enquanto o atributo desempenho é do tipo decimal, sendo atribuído a ele a nota média do aluno nas atividades obrigatórias. Todos os atributos são normalizados entre -1 e 1. Em seguida, os atributos são concatenados para formar o vetor de entrada.

\subsection{Arquitetura da plataforma ASSIS}

O ASSIS foi construído usando quatro componentes principais, conforme apresenta a Figura 2; plugin, servidor, módulo IA, e interface. O plugin é responsável pela extração de dados tanto do AVA da instituição sob análise, quanto de outras bases de dados que contenham informações importantes pare a predição, caso necessário.

O servidor, desenvolvido em Node, executa o processamento das informações vindas do plugin, executa algumas funções necessárias para a predição da evasão, dos indicadores e das métricas que envolvem os cenários dos tutores, cursos, turmas e alunos, além de fornecer servi ços de armazenamento não apenas dos dados comportamentais dos alunos, mas também seus dados cadastrais. Como banco de dados internos foi utilizado o MongoDB. O servidor é responsável também por dois importantes requisitos de qualidade: interoperabilidade e segurança. A primeira está associada à comunicação e à integração com diversos dispositivos, enquanto a segunda diz respeito à privacidade e proteção dos dados dos usuários, já respeitando a Lei Geral de Proteção de Dados.

O módulo de IA é responsável pela predição da evasão escolar utilizando os algoritmos apresentados na seção anterior e os dados extraídos pelo plugin. Ele foi desenvolvido utilizando a linguagem Python fornecendo um serviço de predição em uma API desenvolvida em Flask. O módulo IA normaliza o vetor de caraterística entre -1 e 1, passando ao modelo carregado esse vetor de atributos e mostra a predição feita com os indicadores e métricas.

Por fim, a interface do usuário é uma parte do sistema construído em Node e usa as estruturas Express, Mongoose e React. Ao fazer login na plataforma ASSIS, o usuário é redirecionado para uma tela com duas opções de interação: a primeira consiste em um chatbot, que permite ao usuário realizar perguntas no formato textual, enquanto a segunda consiste em um dashboard contendo indicadores e métricas de evasão. Em ambos os casos, o usuário pergunta ou recebe informação sobre os cursos em andamento e os cursos que já encerraram, além de previsão de risco de evasão considerando tutores, cursos, turmas e alunos.

Outros conceitos abordados e que foram implementados na plataforma do ASSIS são: disponibilidade e escalabilidade. Disponibilidade diz respeito à possibilidade de 


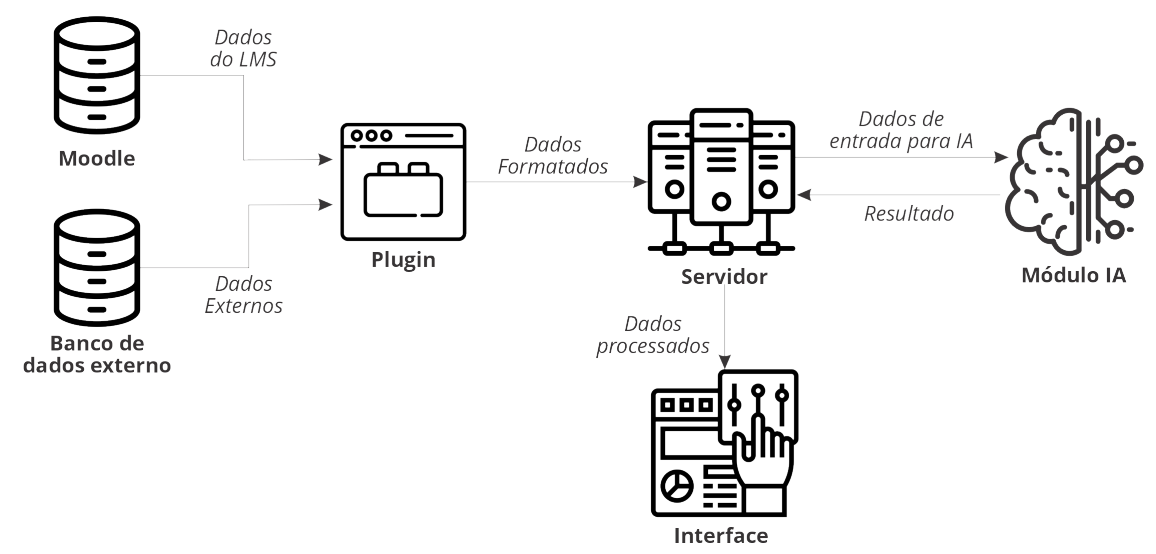

Figura 2. Fluxograma da abordagem proposta para detecção e classificação online de possíveis alunos que evadem do instituto.

acessar o sistema a qualquer momento e utilizando diferentes dispositivos. Caso o ASSIS pare por algum motivo, como queda do servidor, as informações envidadas pelo plugin serão enfileiradas e processadas posteriormente quando o ASSIS se tornar disponível novamente.

Em relação à escalabilidade, é possível que uma grande massa de informações seja processada pelo ASSIS ao mesmo tempo. Para que isso seja possível, ós módulos servidor e de IA do ASSIS são executados na nuvem, tornando o processamento mais eficiente.

\subsection{Interface Gráfica}

O ASSIS possui uma interface gráfica fácil e intuitiva, ilustrada na Figura 3. Essa interface apresenta um dashboard contendo as informações dos cursos em andamento e dos cursos finalizados, sendo possível analisar o histórico do risco de evasão, os indicadores e as métricas que envolvem tutores, cursos, turmas e alunos.

No bloco dos tutores é apresentado o nome de cada tutor, o percentual do risco de evasão de seus alunos, quantos alunos estão em baixo risco de evasão e os que são considerados alto risco de evasão. Nesse bloco, é possível realizar uma pesquisa pelo nome do tutor e selecionar os tutores para comparar as métricas e indicadores entre eles.

No bloco dos cursos é possível identificar o nome do curso, o percentual do risco de evasão do curso, quantos tutores e quantos alunos possuem no curso. Neste bloco, é possível realizar uma busca entre os cursos e selecioná-los para eventuais comparações. O mesmo pode ser aplicado ao bloco das turmas.

Já no bloco dos alunos é possível identificar o nome do aluno, em quantos cursos aquele aluno está matriculado, quantos tutores orientam o aluno e a predição do risco de evasão dos mesmos.

Os blocos do lado esquerdo possibilitam diversas combinações de filtros entre tutores, cursos, turmas e alunos, facilitando ao usuário a entrega dos resultados. A cada filtro aplicado, as informações do lado direito do dashboard são atualizadas, como o histórico do risco de evasão, os indicadores e as métricas.

O histórico do risco de evasão apresenta a quantidade de alunos que estão nas 


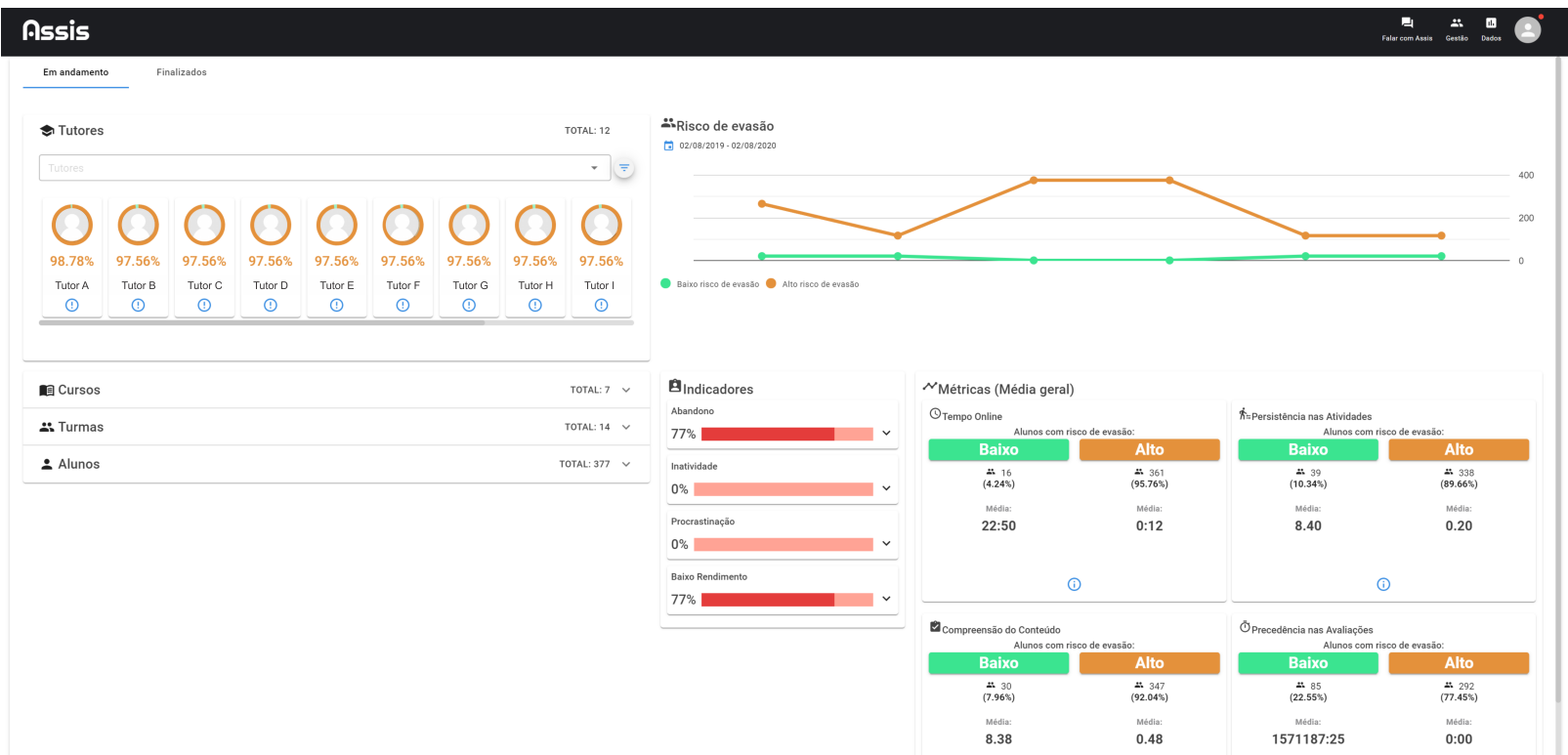

Figura 3. Interface gráfica dashboard

categorias baixo risco e alto risco. Essas informações são atualizadas à medida que novos dados são fornecidos ao módulo IA. Com esse recurso, o usuário pode visualizar o crescimento ou decaimento do risco de evasão dos alunos da plataforma.

Nos indicadores é possível identificar o "retrato falado" dos alunos que podem pertencer a um dos possíveis perfis abordados no ASSIS, sendo eles:

- Abandono: alunos que estão inscritos no curso, mas que não acessaram a plataforma;

- Inatividade: alunos que acessam a plataforma, mas não realizam nenhuma interação;

- Procrastinação: alunos que realizam as atividades nos limites dos prazos;

- Baixo rendimento: alunos que não atingem o mínimo exigido na plataforma.

O usuário poderá navegar entre os perfis e, ao selecionar um perfil, é possível identificar quem são os alunos que pertencem ao perfil selecionado. Com isso, fica clara a identificação da turma, curso e tutor que também pertencem a esse perfil.

As métricas do dashboard possibilitam ao usuário identificar quantos alunos estão nas categorias baixo risco e alto risco em relação a tempo, tentativas, precedência e desempenho. Com isso, o gestor poderá intervir de forma mais precisa, alertando o tutor ou o aluno no que realmente necessita ser melhorado ou corrigido.

\subsection{Métricas}

O módulo de IA do ASSIS foi validado usando as métricas Accuracy, F Score, Recall e Precision. As métricas foram baseadas na matriz de confusão, composta por Verdadeiros Positivos (TP), Verdadeiro Negativo (TN), Falso Negativo (FN) e Falso Positivo (FP).

Accuracy (Acc) é uma medida entre os registros classificados corrigidos e o número total de amostras. Acc é definido na Eq. 1 . 
O Recall mede o número de amostras de uma classe que foi corrigida e prevista para pertencer a essa classe. O Recall é definido na Eq. 2 .

Precision quantifica o número de previsões de uma classe que realmente pertencem a essa classe. Precision é definida na Eq. 3 .

F Score é uma medida que calcula a média harmônica de recuperação e precisão. Eq. 4 mostra como o F Score é calculado.

$$
\begin{gathered}
A c c=\frac{T P+T N}{T P+T N+F P+F N} \\
\text { Recall }=\frac{T P}{T P+T N}
\end{gathered}
$$$$
\text { Precision }=\frac{T P}{T P+F P}
$$

$$
F \text { Score }=2 \cdot \frac{\text { Precision } \cdot \text { Recall }}{\text { Precision }+ \text { Recall }}
$$

\section{Resultados}

Nesta seção são apresentados os resultados coletados utilizando os diferentes algoritmos de aprendizagem de máquina já mencionados. O dataset é composto por 5360 registros comportamentais dos alunos na plataforma EaD Moodle da instituição alvo, selecionando as características tempo, persistência, precedência e desempenho. Esse dataset refere-se a 4620 matrículas que concluíram o curso e 740 matrículas que corresponde à evasão nos cursos da modalidade EaD. Para que o treinamento ocorresse de forma justa para ambas as classes, foram selecionados $80 \%$ dos registros da classe minoritária e a mesma quantidade de registros para a classe majoritária. Com isso, foi possível montar um dataset para treino balanceado entre ambas as classes, e os demais registros foram adicionados para o teste dos classificadores. O dataset foi normalizado entre -1 e 1 .

O hardware usado para teste no servidor é um computador com processador Intel Core-i7 com 3,0 GHz, 16 GB de RAM e com 6GB de GPU (Unidade de processamento gráfico). A parametrização dos algoritmos usados, bem como os resultados obtidos, são apresentados nas subseções a seguir.

\subsection{Parametrização dos classificadores}

Todos os algoritmos de aprendizagem de máquina deste artigo passaram por 50 treinamentos e validação cruzada com 10 -folds. Para o algoritmo perceptron é utilizado a taxa de aprendizagem entre 0,01 a 0,5. O RL foi configurado com uma norma L2. Para o $\mathrm{SVM}$, foi utilizado o kernel RBF, com o hiper-parâmetro de $C$ variando de 0,1 a 10 . Ao final foram usados os melhores resultados de cada algoritmo.

\subsection{Comparação dos resultados}

Na Tabela 2 são apresentados os resultados coletados por cada algoritmos de aprendizagem de máquina. Na Tabela 2, é evidente que o SVM obteve os melhores resultados em todas as métricas analisadas. O SVM alcançou 95,84\% na Accuracy que remete ao acerto global entre as duas classes, ou seja, nessa métrica é contabilizada a taxa de acerto entre os concludentes e os evasores. O SVM obteve $95,74 \%$ na métrica $F$ Score, o que significa 
que existe uma harmonia entre as métricas Precision e Recall. Já na métrica Recall o SVM adquiriu 94,78\%. Essa métrica permite identificar o quanto o algoritmo está acertando os registros dos alunos que realmente são da classe dos concludentes, enquanto a métrica Precision que obteve 96,83\% garante a assertividade dos registros que realmente são da classe dos evasores. Os resultados obtidos pelo SVM supera a Regressão Logística, que obteve o segundo melhor resultado, com uma diferença em 3,45\%, 3,40\%, 2,30\% e 4,36\% em Accuracy, F Score, Recall e Precision, respectivamente. Além disso, o SVM e a Regressão Logística foram os únicos algoritmos que obtiveram resultados superiores a 92\% em todas as métricas.

Tabela 2. Comparação dos resultados considerando Accuracy (Acc), F Score, Recall e Precision como métricas de avaliação.

\begin{tabular}{ccccc}
\hline Algoritmo & Acc (\%) & F Score (\%) & Recall (\%) & Precision (\%) \\
Perceptron & $89,02 \pm 0,0117$ & $89,91 \pm 0,0108$ & $89,90 \pm 0,0156$ & $90,08 \pm 0,0224$ \\
RL & $92,39 \pm 0,0112$ & $92,39 \pm 0,0112$ & $92,48 \pm 0,0026$ & $92,47 \pm 0,0020$ \\
SVM & $\mathbf{9 5 , 8 4} \pm \mathbf{0 , 0 0 8 9}$ & $\mathbf{9 5 , 7 9} \pm \mathbf{0 , 0 0 2 0}$ & $\mathbf{9 4 , 7 8} \pm \mathbf{0 , 0 0 3 3}$ & $\mathbf{9 6 , 8 3} \pm \mathbf{0 , 0 0 2 2}$ \\
\hline
\end{tabular}

Comparando com os demais algoritmos de aprendizagem de máquina, o perceptron não apresentou os melhores resultados, ficando abaixo de $90 \%$ em todas as métricas. No entanto, esse algoritmo não é a melhor opção para tratar os dados apresentados.

Uma outra métrica que pode ser considerada crítica em plataformas que possuem recursos de aprendizagem de máquina é o fator tempo, tanto no treinamento como no teste. Em sistemas preditivos, essa métrica pode se tornar o principal problema devido aos inúmeros dados que podem ser acessados pelos mais diversos dispositivos. A tabela 3 apresenta o tempo realizado para o treinamento e o tempo médio para o teste do vetor de características. Como apresentado em outras métricas, o SVM obteve melhores resultados, significando que ele apresentou menores tempo de treinamento e de teste.

\begin{tabular}{l} 
Tabela 3. Comparação do treinamento dos algoritmos e tempo méd \\
do registro $(\tau)$ em segundos. \\
\cline { 2 - 4 } \\
\begin{tabular}{cccc} 
Algoritmo & Tempo treinamento & $\tau$ \\
\hline Perceptron & $0,437 \pm 0,026$ & $0,003 \pm 0,003$ \\
RL & $0,325 \pm 0,028$ & $0,003 \pm 0,000$ \\
SVM $(\mathrm{RBF})$ & $0,278 \pm 0,033$ & $0,003 \pm 0,000$ \\
\hline
\end{tabular}
\end{tabular}

Considerando o SVM, os parâmetros Accuracy, F Score, Recall e Precision obtiveram os resultados 95,84\%, 95,79\%, 94,78\% e 96,83\%, respectivamente. Isso significa que obteve-se um resultado confiável no processo preditivo de alunos com características de evasão escolar.

\section{Conclusão}

Este artigo apresentou o ASSIS, uma ferramenta para detectar e predizer alunos em risco de evasão escolar com base em seus dados comportamentais extraídos da plataforma EaD utilizada pela instituição. O ASSIS foi planejado para permitir uma interação simples e 
intuitiva de gestores educacionais por meio de infográficos que expressam as métricas e indicadores de evasão e de um chatbot que responde perguntas textuais sobre os riscos de evasão relativos a cursos, turmas, tutores e alunos. Os algoritmos de aprendizado de máquina foram utilizados para encontrar a possibilidade da evasão escolar, oferecendo oportunidades para os gestores e tutores das escolas intervirem com as ações necessárias para reter uma possível evasão escolar. Como demonstrado, o SVM pode ser considerado como uma alternativa viável para realizar esta ação preditiva, uma vez que obteve os melhores resultados, atingindo $95,84 \%$ na Accuracy, $95,79 \%$ no $F$ Score, $94,78 \%$ no Recall e 96,83\% de Precision.

Para trabalhos futuros, pretendemos expandir o ASSIS para que outros dados comportamentais nas plataformas EaD também possam ser incorporados. Além disso, pretende-se aplicar a mesma abordagem a outras instituições. Por fim, espar-se fazer uma análise real de como o ASSIS ajudou a reter o aluno nas instuições que o utilizarem.

\section{Referências}

Al-Shabandar, R., Hussain, A., Laws, A., Keight, R., Lunn, J., and Radi, N. (2017). Machine learning approaches to predict learning outcomes in massive open online courses. In 2017 International Joint Conference on Neural Networks (IJCNN), pages 713-720. IEEE.

Beltran, C. A. R., Xavier-Júnior, J. C., Barreto, C. A., and Neto, C. O. (2019). Plataforma de aprendizado de maquina para detecção e monitoramento de alunos com risco de evasão. In Brazilian Symposium on Computers in Education (Simpósio Brasileiro de Informática na Educação-SBIE), volume 30, page 1591.

Dourado Jr, C. M., da Silva, S. P. P., da Nóbrega, R. V. M., Barros, A. C. d. S., Rebouças Filho, P. P., and de Albuquerque, V. H. C. (2019). Deep learning iot system for online stroke detection in skull computed tomography images. Computer Networks, 152:25-39.

Guenther, N. and Schonlau, M. (2016). Support vector machines. The Stata Journal, 16(4):917-937.

Gütl, C., Rizzardini, R. H., Chang, V., and Morales, M. (2014). Attrition in mooc: Lessons learned from drop-out students. In International workshop on learning technology for education in cloud, pages 37-48. Springer.

Haykin, S. (2008). Neural networks and learning machines. prentice hall. New York.

Martinho, V. R., Nunes, C., and Minussi, C. R. (2013). Prediction of school dropout risk group using neural network. In 2013 Federated Conference on Computer Science and Information Systems, pages 111-114. IEEE.

Menard, S. (2002). Applied logistic regression analysis, volume 106. Sage.

Ramos, J. L. C., Silva, J., Prado, L., Gomes, A., and Rodrigues, R. (2018). Um estudo comparativo de classificadores na previsão da evasão de alunos em ead. In Brazilian Symposium on Computers in Education (Simpósio Brasileiro de Informática na Educação-SBIE), volume 29, page 1463.

Shiratori, N. (2017). Modeling dropout behavior patterns using bayesian networks in small-scale private university. In 2017 6th IIAI International Congress on Advanced Applied Informatics (IIAI-AAI), pages 170-173. IEEE.

Xing, W. and Du, D. (2019). Dropout prediction in moocs: Using deep learning for personalized intervention. Journal of Educational Computing Research, 57(3):547570 . 